- Additional material is published online only. To view please visit the journal online (http://dx.doi.org/10.1136/ neurintsurg-2018-013834).

For numbered affiliations see end of article.

Correspondence to Dr Urs Fischer, Department of Neurology, University of Bern, Bern 3012, Switzerland; urs. fischer@insel.ch

Received 7 February 2018 Revised 26 March 2018 Accepted 3 April 2018 Published Online First 15 May 2018
Check for updates

To cite: Kaesmacher J, Mordasini P, Arnold M, et al. J Neurolntervent Surg 2019:11:20-27.

\title{
Direct mechanical thrombectomy in tPA-ineligible and -eligible patients versus the bridging approach: a meta-analysis
}

\author{
Johannes Kaesmacher, ${ }_{1}^{1,2}$ Pasquale Mordasini, ${ }^{2}$ Marcel Arnold, ${ }^{1}$ Elena López-Cancio, ${ }^{3}$ \\ Neus Cerdá, ${ }^{4}$ Tobias Boeckh-Behrens, ${ }^{5}$ Justus F Kleine, ${ }_{1}^{6}$ Mayank Goyal, ${ }_{1}^{7}$ \\ Michael D Hill, ${ }^{8}$ Vitor Mendes Pereira, ${ }^{9}$ Jeffrey L Saver, ${ }_{1}^{10}$ Jan Gralla, ${ }^{2}$ Urs Fischer ${ }^{1}$
}

\begin{abstract}
Background Whether pretreatment with intravenous thrombolysis prior to mechanical thrombectomy (IVT+MTE) adds additional benefit over direct mechanical thrombectomy (dMTE) in patients with large vessel occlusions (LVO) is a matter of debate.

Methods This study-level meta-analysis was presented in accord with the Preferred Reporting Items for Systematic Reviews and Meta-Analyses guidelines. Pooled effect sizes were calculated using the inverse variance heterogeneity model and displayed as summary Odds Ratio (sOR) and corresponding 95\% confidence interval $(95 \% \mathrm{Cl})$. Sensitivity analysis was performed by distinguishing between studies including dMTE patients eligible for IVT (IVT-E) or ineligible for IVT (IVT-IN). Primary outcome measures were functional independence (modified Rankin Scale $\leq 2$ ) and mortality at day 90, successful reperfusion, and symptomatic intracerebral hemorrhage.
\end{abstract}

Results Twenty studies, incorporating 5279 patients, were included. There was no evidence that rates of successful reperfusion differed in dMTE and IVT+MTE patients (sOR $0.93,95 \% \mathrm{Cl} 0.68$ to 1.28). In studies including IVT-IN dMTE patients, patients undergoing dMTE tended to have lower rates of functional independence and had higher odds for a fatal outcome as compared with IVT+MTE patients (sOR $0.78,95 \% \mathrm{Cl}$ 0.61 to 1.01 and sOR $1.45,95 \% \mathrm{Cl} 1.22$ to 1.73 ). However, no such treatment group effect was found when analyses were confined to cohorts with a lower risk of selection bias (including IVT-E dMTE patients). Conclusion The quality of evidence regarding the relative merits of IVT+MTE versus dMTE is low. When considering studies with lower selection bias, the data suggest that dMTE may offer comparable safety and efficacy as compared with IVT+MTE. The conduct of randomized-controlled clinical trials seems justified.

\section{INTRODUCTION}

Seven randomized-controlled studies have consistently shown that patients with a large vessel occlusion (LVO) in the anterior circulation benefit from mechanical thrombectomy (MTE) following intravenous thrombolysis (IVT) with tPA. ${ }^{1-7}$ Currently it is unclear if IVT before MTE adds additional benefit ${ }^{8-11}$ and this question will be assessed in two randomized-controlled studies (SWIFT DIRECT,
MR CLEAN NO IV). The individual patient data meta-analysis of the pivotal thrombectomy trials found no interaction between the treatment effect size of MTE and IVT pretreatment status ${ }^{12}$ and a recently published study-level meta-analysis found no significant difference in outcome between patients with and without pretreatment with IVT. ${ }^{13}$ In contrast, another meta-analysis has suggested that IVT + MTE patients had better functional outcomes, lower mortality, higher rates of successful recanalization, and equal odds of symptomatic intracerebral hemorrhage compared with patients treated with direct MT (dMTE). ${ }^{14}$ However, the aforementioned analyses may in part be confounded by the eligibility for IVT, potentially leading to group imbalances in stroke etiology, risk factors, and time to treatment.

To account for such group imbalances more systematically, the present meta-analysis on a comparison of IVT + MTEversus dMTE will distinguish between studies including dMTE patients eligible for IVT (IVT-E) and studies including dMTE patients ineligible for IVT (IVT-IN), as the latter may be a major source of selection bias. In addition, baseline risk factor distribution in both groups will be evaluated to quantify selection bias.

\section{METHODS}

This study-level meta-analysis was presented in a manner adherent to the Preferred Reporting Items for Systematic Reviews and Meta-Analyses (PRISMA) guidelines. ${ }^{15}$ The checklist outlined by the Meta-analysis Of Observational Studies in Epidemiology (MOOSE) consortium was also applied. The authors declare that all supporting data are available within the article and its online supplementary files. Extracted raw data can be found in the online supplementary dataset 1 .

\section{Search strategy and selection criteria}

Information about the search strategy and accessed databases can be found as additional information in the Online Supplement (supplementary additional information 1 and table I-IV).

All identified studies were imported into EndNote (version X, Clarivate Analytics, Phiadelphia, USA) and an automatic deduplication was performed. After the deduplication procedure, studies were reviewed for eligibility concerning the quantitative 
analysis by two independent raters (JK, PM) and by a third rater in cases of discrepancies (UF). Prespecified inclusion criteria can be found in the Online Supplement (supplementary additional information 2).

Studies were categorized as comparing dMTE in IVT-eligible patients (=IVT-E) with IVT+MTE in IVT-eligible patients or comparing dMTE in IVT-ineligible patients (=IVT-IN) with IVT + MTE in IVT-eligible patients. Studies reporting on both IVT-E and IVT-IN patients were included if separate analyses of these groups had been presented in the published full text article, or if the relevant data discriminating between the two groups were made available after contacting the respective corresponding authors. If a study provided comparison of a matched and unmatched control group, the matched-pair analysis was used for further calculation in order to reduce the risk of bias. Any matched-pair analysis was excluded from the analyses regarding risk-factor distribution between dMTE and IVT+MTE patients.

\section{Extraction of baseline and outcome data}

Data were extracted by JK according to a standardized extraction sheet and cross-checked by a second reader. A full list with the study data and outcomes extracted, as well as contact processes with the authors can be found as additional information in the Online Supplement (supplementary additional information 3).

\section{Statistical analysis and quality assessment}

Pooled effect sizes were calculated using the inverse variance heterogeneity (IVhet) model, assuming heterogeneous studies and the presence of multiple true effect sizes. ${ }^{16}$ The assumption was based on the heterogeneous inclusion and exclusion criteria of the respective studies. The IVhet model is considered as an improved alternative to the random effect model which uses an estimator under the fixed effect model assumption with a quasi-likelihood based variance structure. ${ }^{16}$ For all outcomes included in the analysis, we calculated the corresponding summary ORs and respective 95\% CIs to express the comparison of dMTE vs IVT+MTE.

Pooled effect sizes were further calculated and stratified according to IVT-E and IVT-IN studies. Other subgroup analyses were performed according to the study type (ie, confined analysis to RCT subgroup analyses). For further information regarding influence analysis, assessment of heterogeneity and evaluation of publication bias, see Online Supplement (supplementary additional information 4). Quality assessment was performed as denoted in the Online Supplement (supplementary additional information 5). Data were analyzed and displayed using the software package MetaXL (EpiGear International, Sunrise Beach, Queensland, Australia) for Microsoft Excel.

\section{RESULTS}

\section{Literature search}

The search strategies yielded 2747 unique publications, among which 2617 were excluded on abstract and title screening. After retrieving the full texts of the remaining 130 studies, 20 met all study selection criteria and were included into quantitative synthesis (see online supplementary figure 1 for literature search flow diagram, table 1 for study characteristics). ${ }^{15}{ }^{17-33}$ Reporting rates of the primary and secondary outcomes are denoted in online supplementary table V. Further information regarding the results of the literature search is provided in the Online Supplement (supplementary additional information 6).

\section{Baseline characteristics and risk factor distribution}

For detailed information see Supplementary additional information 7. In short, IVT-IN dMTE patients were treated later and had more comorbidities including higher odds for a medical history of atrial fibrillation (sOR 1.94 , 95\% CI 1.50 to 2.50, online supplementary figure IV) and prior cerebrovascular events (sOR $1.94,95 \%$ CI 1.60 to 2.35 , online supplementary figure IV).

\section{Functional independence at day 90}

Rates of functional independence at 3 months were reported in 17 studies (2 IVT-E, 1 IVT-E, and IVT-IN, 14 IVT-IN, 4657 patients). Overall, there was a non-significant trend that patients undergoing dMTE have lower rates of functional independence at day 90 as compared with IVT+MTE patients (sOR 0.82, 95\% CI: 0.65 to 1.03 , figure 1 ). However, heterogeneity was substantial $\left(\mathrm{I}^{2}\right.$ : $\left.51 \%, \mathrm{Q}=35.01, \mathrm{P}=0.01\right)$. Influence analysis provided evidence that the exclusion of three single studies (Weber et al, ${ }^{19}$ Abilleira et $\mathrm{al}^{21}{ }^{21}$ and Sanak et $\mathrm{al}^{31}$ ) would result in a significant association between dMTE and lower rates of good functional outcome (data not shown). Analysis confined to RCTs, comparing dMTE in IVT-IN patients and IVT+MTE in IVT-E patients, showed no differences between the groups, as evidenced by a point estimation of sOR 0.80 (95\% CI 0.58 to 1.12 , online supplementary figure VII).

Among just the studies comparing dMTE in IVT-E patients with IVT+MTE in IVT-E patients, rates of functional independence at 3 months did not differ between the treatment groups (sOR $1.08,95 \%$ CI 0.67 to 1.76 , figure 1 ).

\section{Mortality at day 90}

Rates of mortality at day 90 were reported in 18 studies (2 IVT-E, 1 IVT-E/IN, and 15 IVT-IN studies, 4929 patients). Patients undergoing dMTE had higher odds for a fatal outcome at day 90 (sOR $1.35,95 \%$ CI 1.07 to 1.71 , figure 2). No substantial heterogeneity was noted, with $39 \%, 27.70$, and $\mathrm{P}=0.05$ for $\mathrm{I}^{2}, \mathrm{Q}$, and corresponding $\mathrm{p}$, respectively. Influence analysis provided evidence that the exclusion of one study (Minnerup et $\mathrm{al}^{26}$ ) resulted in loss of significance (data not shown). Restriction to RCT data, comparing dMTE in IVT-IN patients and IVT + MTE in IVT-E patients, provided a comparable point estimate as other IVT-IN studies (sOR 1.83, 95\% CI 1.16 to 2.91, online supplementary figure VIII).

In the analysis confined to studies comparing dMTE in IVT-E patients with IVT + MTE in IVT-E patients, no effect of treatment group on mortality was noted (sOR $0.84,95 \%$ CI 0.40 to 1.75 , figure 2 ).

\section{Successful reperfusion}

Nineteen studies reported on rates of successful reperfusion of IVT+MTEversus dMTE (2 IVT-E, 1 IVT-E, and IVT-IN, 16 IVT-IN, 4220 patients). Overall, there was no evidence that rates of successful reperfusion differed in AMTE and IVT + MTE patients (sOR $0.93,95 \% \mathrm{CI} 0.68$ to 1.28 , figure 3 ). $\mathrm{I}^{2}$ and Cochrances $Q$ statistics were $\mathrm{I}^{2}=51 \%$ (95\% CI: $19 \%$ to $71 \%$ ) and $\mathrm{Q}=39.02, \mathrm{P}<0.01$, indicating substantial heterogeneity. Influence analysis did not provide evidence that the sOR changed significantly when excluding single studies (data not shown). Similar rates of successful reperfusion were also observed when the analysis was confined to analyses of RCTs with dMTE in IVT-IN patients and IVT+MTE in IVT-E patients (sOR 1.32, 95\% CI 0.89 to 1.95 , online supplementary figure IX). Among nine studies (1753 patients) reporting rates of complete (TICI3) 


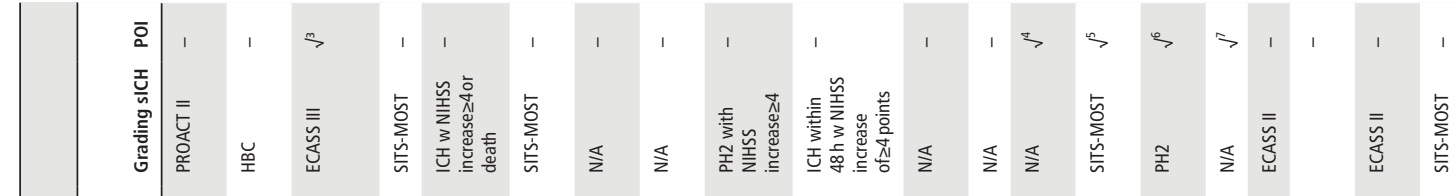

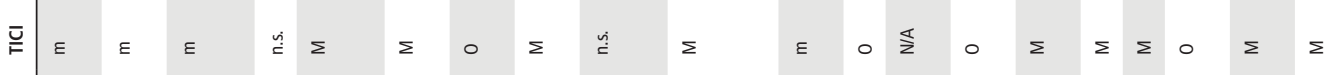

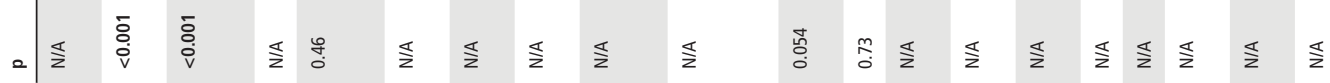

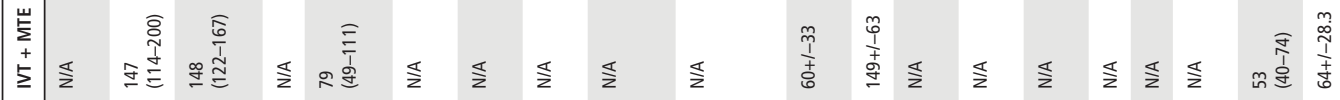

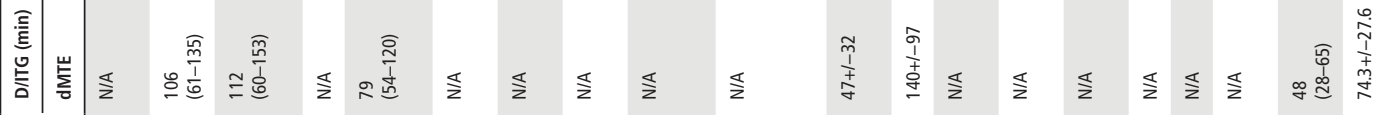

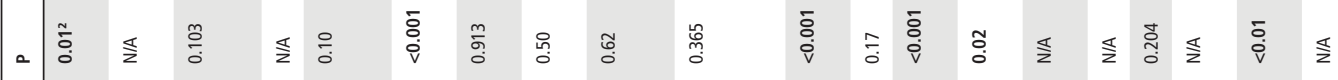

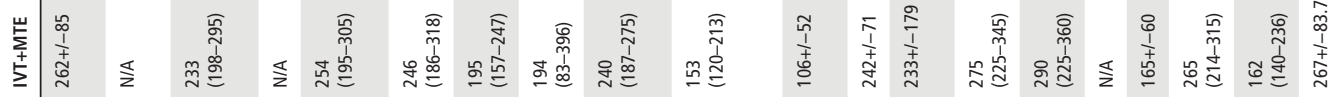

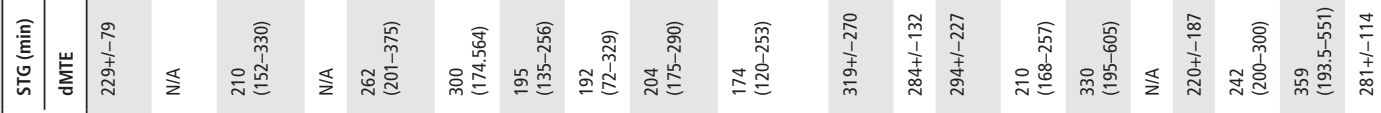

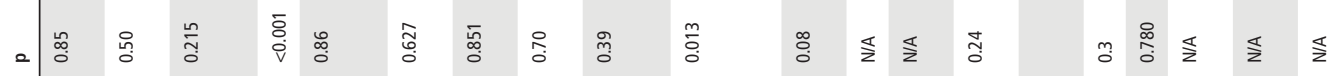

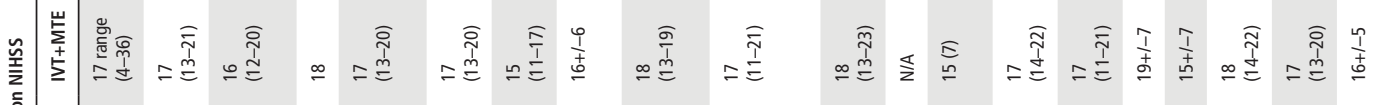

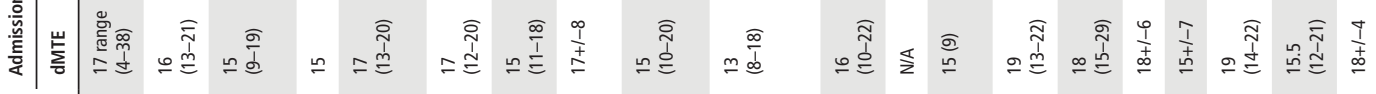

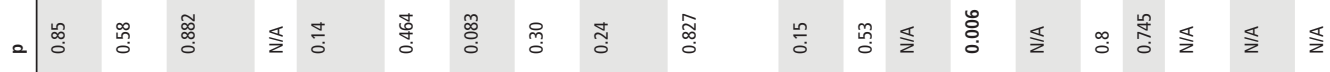

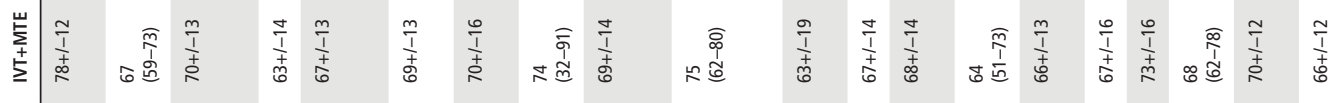

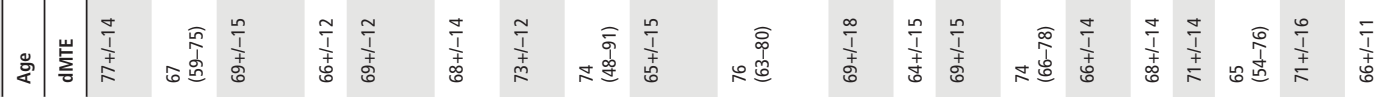 岀䓬

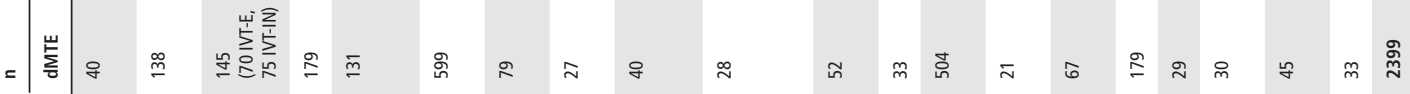

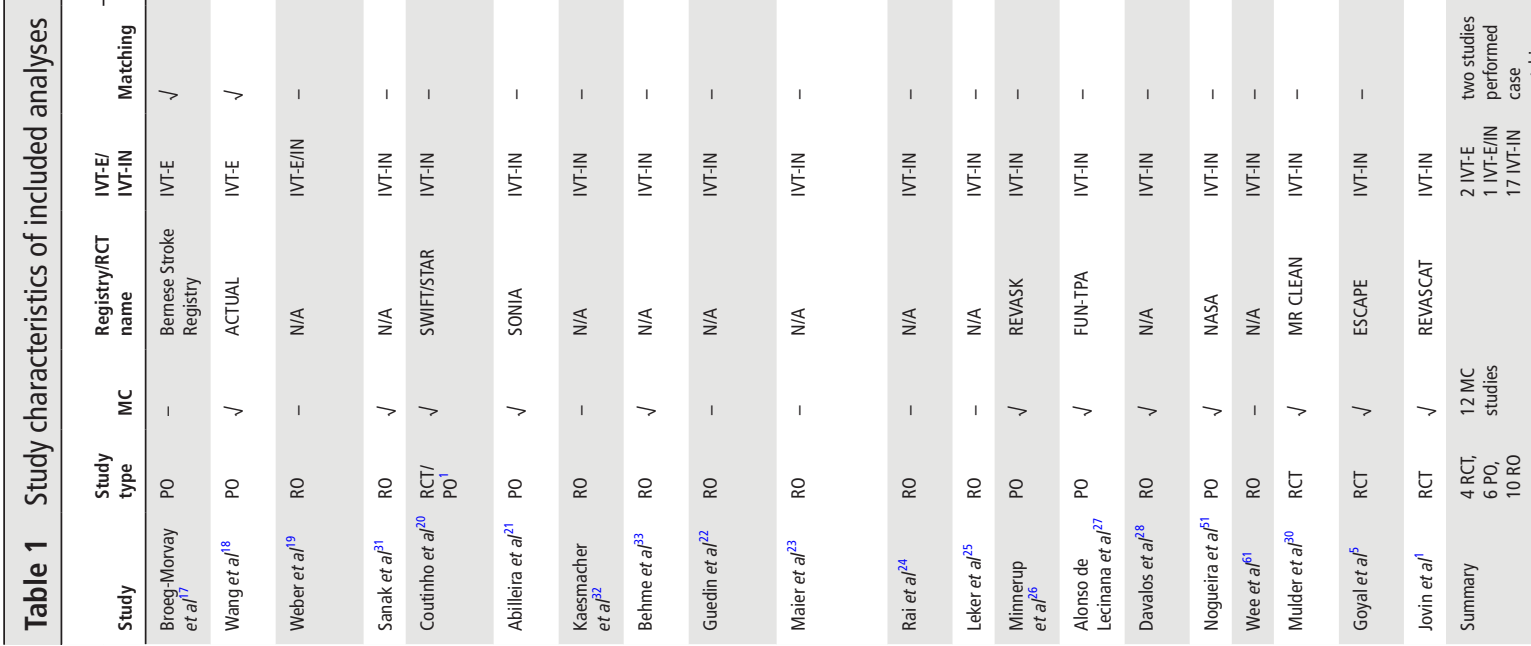




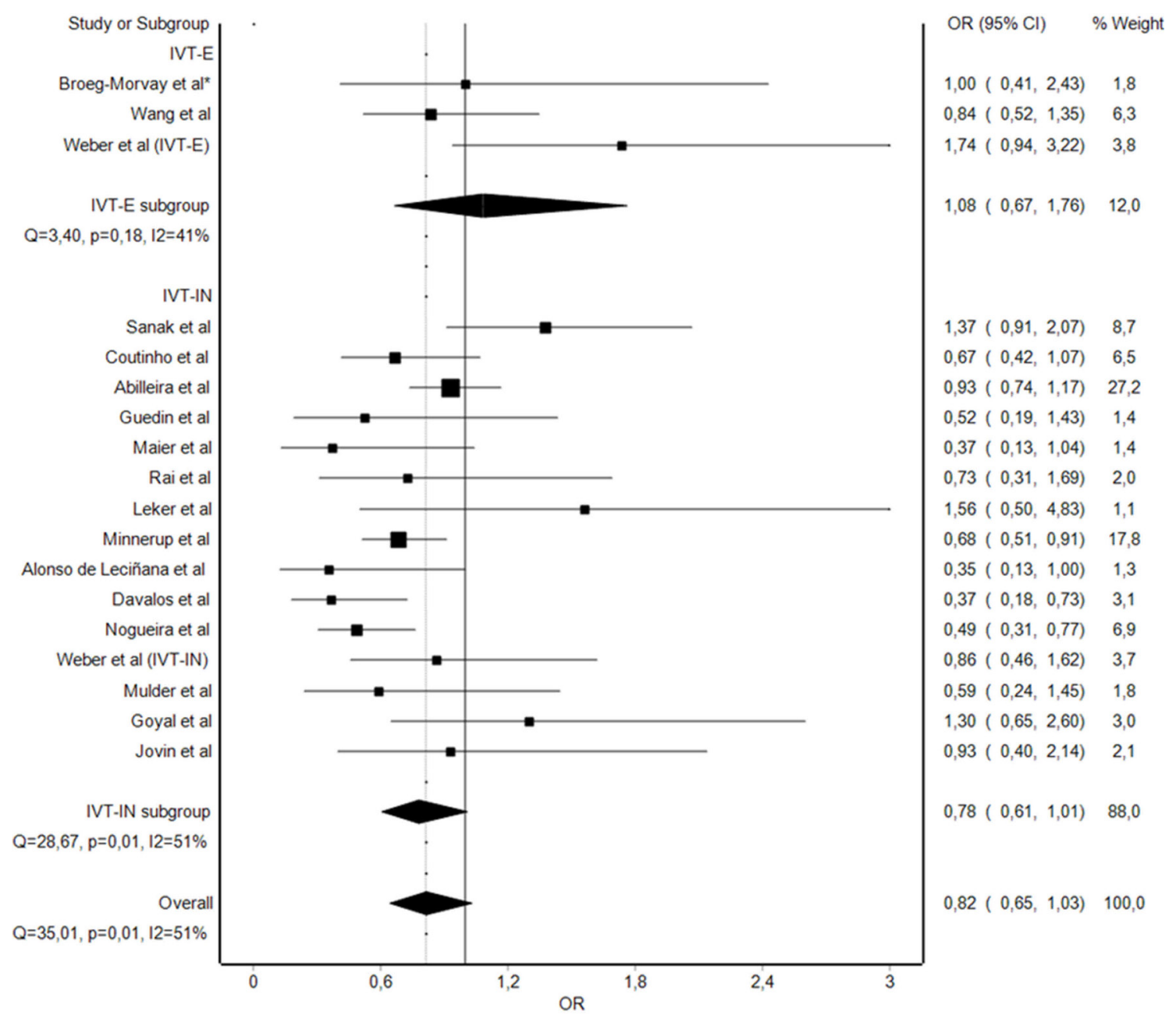

Favors IVT+MTE

Favors dMTE

Figure 1 Summary OR of dMTE patients for day $90 \mathrm{mRS} \leq 2$. * matched-pair analysis; IVT-E, IVT-eligible dMTE patients; IVT-IN, IVT-ineligible dMTE patients.

reperfusion, no difference between $\mathrm{dMTE}$ and IVT $+\mathrm{MTE}$ patients was noted (sOR $0.98,95 \%$ CI 0.80 to 1.21 , see online supplementary figure $\mathrm{X})$.

When assessing the rates of successful reperfusion only for studies comparing dMTE in IVT-E patients with IVT+MTE in IVT-E patients ( $\mathrm{n}=531$ patients), there was a non-significant trend for higher rates of successful reperfusion in the dMTE group (sOR $1.67,95 \%$ CI 0.95 to 2.94 , figure 3 ).

\section{Symptomatic intracerebral hemorrhage}

Rates of sICH were reported in 16 studies (3903 patients, see table 1 for respective definition of sICH in the different studies). IVT+MTE patients nominally had more sICH (sOR $0.86,95 \%$ CI 0.63 to 1.17 , figure 4 ) without reaching statistical significance.

Considering only the studies comparing dMTE in IVT-E patients with IVT + MTE in IVT-E patients, no treatment group effect on sICH rates was noted (sOR $0.95,95 \%$ CI 0.51 to 1.76 , figure 4).

\section{Asymptomatic intracerebral hemorrhage}

The reporting frequency for aICH was 75\% (15/20 studies, 3635 patients). Two IVT-E, 12 IVT-IN studies and one IVT-E/IN study had analyzable data. The odds for aICH did not differ between
dMTE and IVT+MTE patients (sOR $0.93,95 \%$ CI 0.72 to 1.19 online supplementary figure XI). The observed heterogeneity was low overall.

However, in the analysis restricted to studies comparing dMTE in IVT-E patients with IVT+MTE in IVT-E patients, dMTE was associated with lower rates of aICH (sOR $0.49,95 \%$ CI 0.30 to 0.81 , online supplementary figure XI).

\section{Quality and bias assessment}

Risk of bias was noted in all studies, with the most common source being nonrandom allocation to treatment groups, present in all. Studies differed in sources of potential bias most often with regard to: blinding of outcome assessment; comparability of baseline characteristics between treatment groups; and non-reporting of whether some patients not undergoing MTE after IVT did so because of preinterventional reperfusion or because of neurological improvement or other reasons (intention to treat reporting, see online supplementary table VII). A minor to moderate funnel and doi plot asymmetry was present for most of the analyses performed (see online supplementary figure XII).

\section{DISCUSSION}

A substantial body of observational and indirect RCT comparison investigations analyzing direct mechanical thrombectomy 


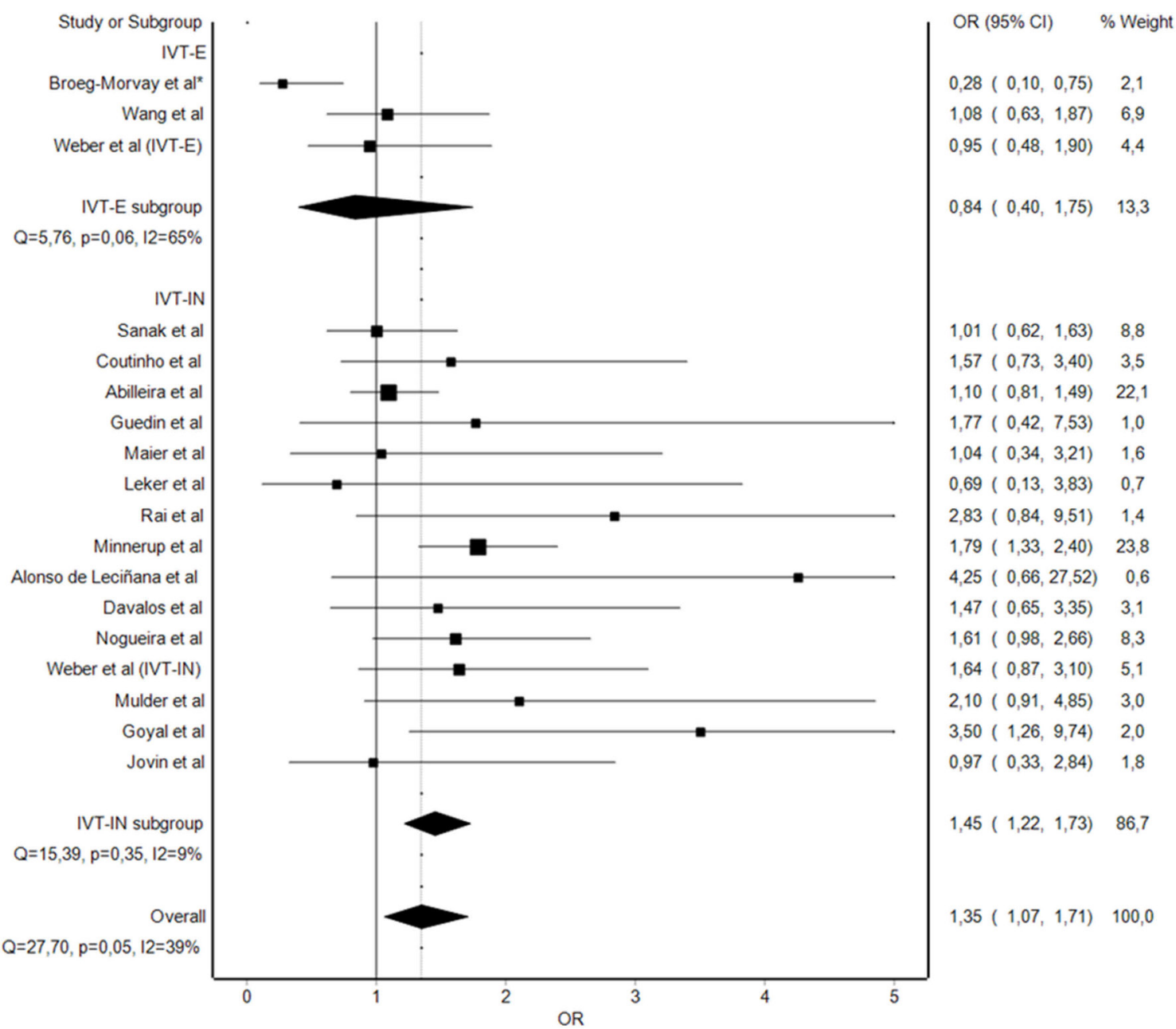

Favors dMTE

Favors IVT+MTE

Figure 2 Summary OR of dMTE patients for fatal outcome at day 90. *matched-pair analysis; IVT-E, IVT-eligible dMTE patients; IVT-IN, IVT-ineligible dMTE patients.

alone vs combined intravenous tPA plus mechanical thrombectomy has now been accumulated, comprising 20 studies and 5279 patients. Intriguingly, in the pooled analysis of three of the studies contrasting both treatment strategies in highly comparable patients - those eligible for IV tPA - direct mechanical thrombectomy alone showed equal rates of substantial reperfusion and lower rates of asymptomatic hemorrhagic transformation, though no differences in 3-month functional independence and mortality. Treatment strategy effects were less consistent when these data were combined with studies contrasting both treatment strategies in less comparable patients (patients with dMTE who were ineligible for IVT). In these patients, dMTE compared with IVT+MTE was associated with a tendency for less symptomatic hemorrhage but higher 3-month mortality.

\section{Comparison with previous meta-analyses}

The findings of the present systematic analysis are consonant by definition with prior component studies, as the meta-analysis encompasses all identified individual investigations. Our findings do however contrast with those of a recent meta-analysis. ${ }^{14}$ That study reported lower reperfusion rates and inferior clinical outcomes among dMTE compared with IVT+MTE patients. ${ }^{14}$ However, that analysis was confined to a smaller group of studies (13 instead of 20), and included only one of the three studies with comparable, IVT-eligible patients in both treatment arms. In contrast, another meta-analysis performed by Phan et $\mathrm{al}^{13}$ showed equal rates of good functional outcome and 90-day mortality between dMTE and IVT+MTE patients. These discrepant results may partially be due to different inclusion criteria, as Phan et $\mathrm{al}^{13}$ also included studies evaluating posterior circulation strokes and a few studies which included first-generation mechanical thrombectomy devices. ${ }^{134}$ Another reason which may partially explain these discrepancies is the difference with regards to the inclusion of studies assessing dMTE in IVT-eligible patients. ${ }^{13} 14$ The current, meta-analysis suggests that, among IVT-eligible patients, dMTE is associated with equal rates of good angiographic results and may protect from asymptomatic hemorrhagic transformation. Hence, some reassurance is provided that reperfusion rates are unlikely to be substantially lower for dMTE patients. The results suggests caution in reaching conclusions from comparisons of dMTE in IVT-ineligible patients with IVT +MTE in IVT-eligible patients. The features that cause patients to be IVT-ineligible also often predispose to lesser technical and clinical success. Those factors may include greater time since onset (allowing more thrombus impaction), recent surgery (greater co-morbidities), and anticoagulation therapy at onset (increased hemorrhagic risk). The current analysis fails to provide evidence for clear benefits of one treatment approach over the other but rather points toward clinical equipoise regarding the best treatment strategy and wariness 


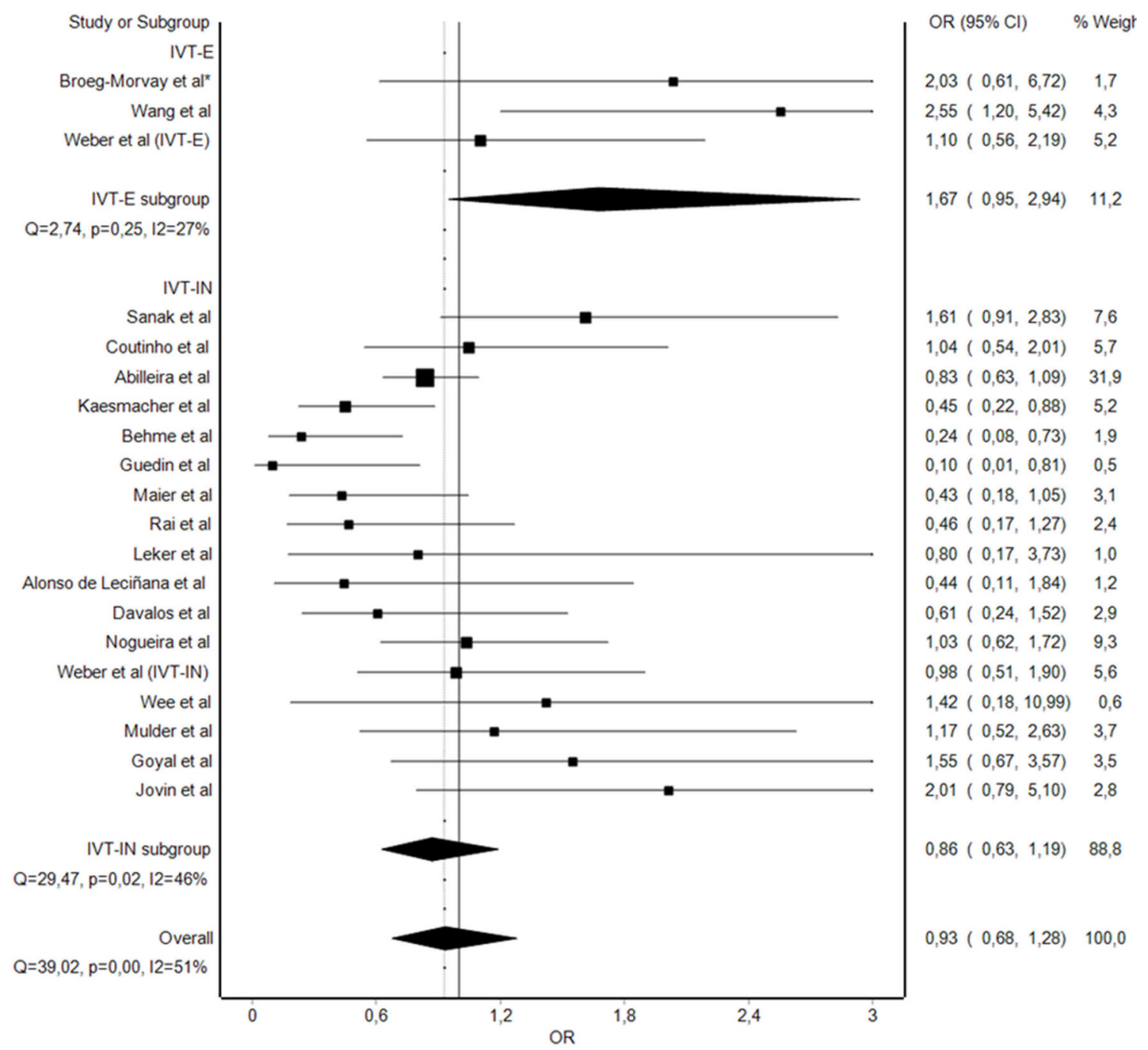

Favors IVT+MTE

Favors dMTE

Figure 3 Summary OR of dMTE patients for successful reperfusion. *matched-pair analysis; IVT-E, IVT-eligible dMTE patients; IVT-IN, IVT-ineligible dMTE patients.

regarding comparison of unlike patients, indicating a need for formal testing in randomized-controlled trials.

\section{Reperfusion}

Numerous theoretical considerations have been proposed supporting both potentiation of MTE reperfusion by preceding IVT (eg, thrombus softening and lysis of downstream emboli), ${ }^{35}$ and reduction of MTE reperfusion by preceding IVT (eg, thrombus fragmentation and procedure delay with further clot organization). ${ }^{36}$ We analyzed rates of complete (TICI 3) as well as successful (TICI $2 \mathrm{~B} / 3$ ) reperfusion, as multiple studies have suggested that complete reperfusion is associated with better clinical outcomes than moderately successful reperfusion. ${ }^{37-42}$ As it is conceivable that IV fibrinolysis might distinctively clear small distal thrombi, ${ }^{35}$ enabling complete rather than moderately successful reperfusion, this topic is of interest. However, in concordance with the findings regarding successful reperfusion, no difference was noted in rates of complete reperfusion (TICI3) between dMTE and IVT+MTE strategies.

\section{Functional outcome}

Important confounding factors were observed in the analysis comparing dMTE in IVT-ineligible patients with IVT+MTE in IVT-eligible patients. The IVT-ineligible patients had worse baseline profiles, including more frequent history of atrial fibrillation, more frequent history of prior cerebrovascular events, and longer total ischemia time prior to start of the thrombectomy procedure. Patients with ischemic stroke and history of atrial fibrillation (AF), compared with non-AF patients, have larger cerebral infarcts, more severe presenting deficits, more frequent post-stroke medical and neurological complications, and worse long-term clinical outcomes. ${ }^{43-45}$ Especially when favorable outcome is evaluated with the modified Rankin Scale, stroke outcomes have been shown to be dependent on preexisting comorbidities such as prior strokes. ${ }^{46}$ Finally, longer time to treatment start is a substantial risk factor for worsened clinical outcome. ${ }^{47}$ Importantly, no difference in rates of good functional outcome were noted when analyses was limited to dMTE patients who were IVT-eligible. This observation was also shared in a patient-level pooled analyses of the data from two articles included in the IVT-eligible subgroup. ${ }^{11}$

\section{Hemorrhagic complications}

Hemorrhagic transformation is a feared complication of cerebral ischemia associated with high morbidity and mortality. ${ }^{48}$ In patients treated with MTE, occurrence of intracranial 


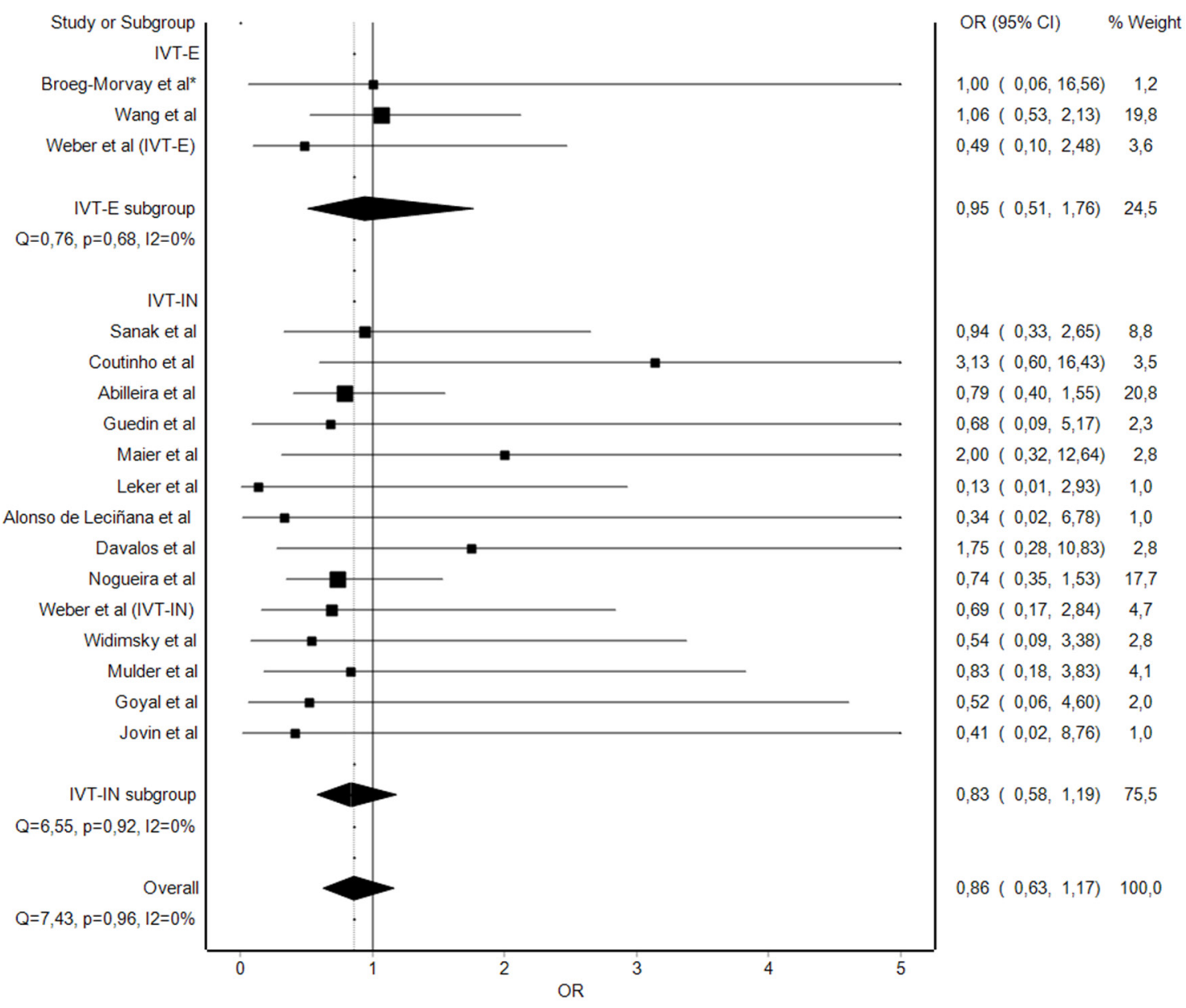

Favors dMTE

Favors IVT+MTE

Figure 4 Summary OR of dMTE patients for symptomatic intracerebral hemorrhage. *matched-pair analysis; IVT-E, IVT-eligible dMTE patients; IVTIN, IVT-ineligible dMTE patients.

hemorrhage is favored by delayed treatment, a proximal occlusion site, cardioembolic origin, poor collaterals, reperfusion success, and extended early ischemic damage. ${ }^{49-52}$ In patients not treated with MTE, IV tPA facilitates the occurrence of intracranial hemorrhage as compared with placebo. ${ }^{53} 54$ In the present meta-analysis, we found signals - albeit weak - that IV tPA added to MTE, compared with MTE alone, increases the likelihood of hemorrhagic transformations.

\section{LIMITATIONS}

This study has several important limitations. First, all studies included in the meta-analysis had potential sources of bias, limiting the strength of conclusions. Second, heterogeneity between studies was noted for some analyses. The use of a conservative statistical approach (inverse variance heterogeneity model $)^{24}$ and of sensitivity analyses dropping individual studies mitigated, but presumably could not entirely account for, heterogeneity effects. Third, we implemented the data of two studies, which performed case-matching in order to reduce the risk of bias owning to differences in baseline characteristics. However, case-matching may also increase the risk of hidden bias and may thus produce artificial results. ${ }^{25}$ Fourth, we did not publish an analysis protocol ahead of the initiation of the meta-analysis. Last, the most important limitation arises due to the fact that several of the studies did not report on patients who were treated with a combined IVT + MTE strategy, but only received the IVT component as they reperfused or clinically improved with IVT alone, before MTE initiation. As a result, this potentially important advantage of the bridging approach was not fully incorporated in the present analysis and deserves future research. Although IVT clearly favors recanalization in patients with LVO, ${ }^{55}$ reperfusion may not occur early enough and reported rates of reperfusions after IVT and prior to the start of MTE are low in patients with an intention to bridge $(\sim 10 \%) .{ }^{13-656-59}{ }^{60}$ Especially non-transfer patients with short needle to groin puncture intervals, a proximal occlusion, and a high thrombus burden are unlikely to respond to IVT. ${ }^{58} 59$ Given these considerations, SWIFT DIRECT (https://clinicaltrials.gov/ ct2/show/NCT03192332) will only include non-transfer patients with a proximal occlusion in whom endovascular treatment can be initiated rapidly.

\section{CONCLUSION}

The overall quality of evidence regarding the relative merits of IVT+MTE versus dMTE is low. In contrast to previous synopses and when analysis is confined to studies with a low risk of selection bias (ie, comparable IVT-eligible patients in both treatment strategy groups), the data suggest that for patients who finally undergo MTE, dMTE may offer comparable safety and efficacy as compared with IVT+MTE. Outcome comparisons yield mixed results when less comparable patients are considered (dMTE in IVT-ineligible patents vs IVT+MTE in IVT-eligible 
patients), but there is evidence of confounding by indication. The available data does provide substantial indications of clinical non-inferiority, suggesting that the conduct of randomized clinical trials evaluating dMTE versus IVT+MTE in LVO patients is appropriate when including only major vessel occlusions and when rapid access to endovascular treatment can be assured. The value of preinterventional recanalization in IVT+MTE needs further evaluation and should be reported more consistently.

\section{Author affiliations}

'Department of Neurology, Inselspital, University Hospital Bern, University of Bern, Bern, Switzerland

2Institute of Diagnostic and Interventional Neuroradiology, Inselspital, University Hospital Bern, University of Bern, Bern, Switzerland

${ }^{3}$ Department of Neurology, Stroke Unit Hospital Universitario Central de Asturias (HUCA), Oviedo, Spain

${ }^{4}$ Biostatistics Unit, Bioclever CRO, Barcelona, Spain

${ }^{5}$ Institute of Diagnostic and Interventional Neuroradiology, Technical University Munich, Klinikum rechts der Isar, München, Germany

${ }^{6}$ Institute of Neuroradiology, Berlin, Germany

${ }^{7}$ Department of Diagnostic Imaging, University of Calgary, Calgary, Alberta, Canada

${ }^{8}$ Department of Clinical Neurosciences, University of Calgary, Calgary, Alberta,

Canada

${ }^{9}$ Division of Neuroradiology, Department of Medical Imaging and Division of Neurosurgery, Department of Surgery, Toronto Western Hospital, University Health Network, Toronto, Ontario, Canada

${ }^{10}$ Comprehensive Stroke Center, Geffen School of Medicine, University of California, Los Angeles, California, USA

Acknowledgements We gratefully acknowledge the contribution of Doris Kopp (Library \& Information Services for the Institutes of Social \& Preventive Medicine (ISPM), Switzerland) for the help in establishing a search strategy.

Contributors All authors contributed to the presented work by substantial contributions to the conception or design of the work; or the acquisition, analysis, or interpretation of data for the work and drafting the work or revising it critically for important intellectual content and final approval of the version to be published and agreement to be accountable for all aspects of the work in ensuring that questions related to the accuracy or integrity of any part of the work are appropriately investigated and resolved.

Funding The work of JK was supported by the SAMW/Bangerter Foundation through the Young Talents in Clinical Research Program. All other authors have not declared a specific grant for this research from any funding agency in the public, commercial, or not-for-profit sectors.

Competing interests Related: UF and JG are global PIs for the SWIFT DIRECT trial and are consultants for Medtronic. VMP is a PI for the SWIFT DIRECT trial and is a consultant for Medtronic. Unrelated: MA received honoraria for lectures and advisory boards from Bayer, Boehringer Ingelheim, Bristol Meyer Squibbs, Pfizer, and Covidien. MG is a consultant for Medtronic, Stryker, Microvention/ and Ablynx and received grants from Medtronic and Stryker provided to the University of Calgary. He has a licensing agreement with GE for systems of stroke diagnosis. MDH received a grant from Alberta Innovates for stroke program in Alberta. VMP is a consultant for Stryker (SC for DAWN trial), Penumbra (SC for PROMISE study), BALT (proctorship of products unrelated to ischemic stroke), Phenox, Rapid Medical, Neurovasc and receives research a grant from Philips. JLS is a consultant about trial design and conduct for Covidien and Stryker, and employee of the University of California, which holds a patent on retriever devices for stroke. JG is a global PI of STAR, CEC member of the PROMISE study (Penumbra), Consultancy; and receives SNSF grants for magnetic resonance imaging in stroke. UF receives research grants from Swiss National Science Foundation (SNSF). All other authors have nothing to disclose.

Patient consent Not required.

Provenance and peer review Not commissioned; externally peer reviewed.

Data sharing statement Raw data and frequency counts can be found by accessing the provided Online Dataset 1.

Open access This is an open access article distributed in accordance with the Creative Commons Attribution Non Commercial (CC BY-NC 4.0) license, which permits others to distribute, remix, adapt, build upon this work non-commercially, and license their derivative works on different terms, provided the original work is properly cited and the use is non-commercial. See: http://creativecommons.org/ licenses/by-nc/4.0/

C) Article author(s) (or their employer(s) unless otherwise stated in the text of the article) 2019. All rights reserved. No commercial use is permitted unless otherwise expressly granted.

\section{REFERENCES}

1 Jovin TG, Chamorro A, Cobo E, et al. Thrombectomy within 8 hours after symptom onset in ischemic stroke. N Engl J Med 2015;372:2296-306.

2 Berkhemer OA, Fransen PS, Beumer D, et al. A randomized trial of intraarterial treatment for acute ischemic stroke. N Eng/ J Med 2015;372:11-20.

3 Campbell BC, Mitchell PJ, Kleinig TJ, et al. Endovascular therapy for ischemic stroke with perfusion-imaging selection. N Engl J Med 2015;372:1009-18.

4 Saver JL, Goyal M, Bonafe A, et al. Stent-retriever thrombectomy after intravenous t-PA vs. t-PA alone in stroke. N Eng/ J Med 2015;372:2285-95.

5 Goyal M, Demchuk AM, Menon BK, et al. Randomized assessment of rapid endovascular treatment of ischemic stroke. N Eng/ J Med 2015;372:1019-30.

6 Bracard S, Ducroca X, Mas JL, et al. Mechanical thrombectomy after intravenous alteplase versus alteplase alone after stroke (THRACE): a randomised controlled trial. Lancet Neurol 2016;15:1138-47.

7 Muir KW, Ford GA, Messow CM, et al. Endovascular therapy for acute ischaemic stroke: the Pragmatic Ischaemic Stroke Thrombectomy Evaluation (PISTE) randomised, controlled trial. J Neurol Neurosurg Psychiatry 2017;88:38-44.

8 Fischer U, Kaesmacher J, Molina CA, et al. Primary Thrombectomy in tPA (Tissue-Type Plasminogen Activator) Eligible Stroke Patients With Proximal Intracranial Occlusions. Stroke 2018:49:265-9.

9 Fischer U, Kaesmacher J, Mendes Pereira V, et al. Direct mechanical thrombectomy versus combined intravenous and mechanical thrombectomy in large-artery anterior circulation stroke: a topical review. Stroke 2017;48:2912-8.

10 Chandra RV, Leslie-Mazwi TM, Mehta BP, et al. Does the use of IV tPA in the current era of rapid and predictable recanalization by mechanical embolectomy represent good value? J Neurointerv Surg 2016;8:443-6.

11 Bellwald S, Weber R, Dobrocky T, et al. Direct mechanical intervention versus bridging therapy in stroke patients eligible for intravenous thrombolysis: a pooled analysis of 2 registries. Stroke 2017;48:3282-8.

12 Goyal M, Menon BK, van Zwam WH, et al. Endovascular thrombectomy after large-vessel ischaemic stroke: a meta-analysis of individual patient data from five randomised trials. Lancet 2016;387:1723-31.

13 Phan K, Dmytriw AA, Maingard J, et al. Endovascular thrombectomy alone versus combined with intravenous thrombolysis. World Neurosurg 2017;108:850-8.

14 Mistry EA, Mistry AM, Nakawah MO, et al. Mechanical thrombectomy outcomes with and without intravenous thrombolysis in stroke patients: a meta-analysis. Stroke 2017:48:2450-6.

15 Moher $\mathrm{D}$, et al. Preferred reporting items for systematic reviews and meta-analyses: the PRISMA statement (Chinese edition). J Chinese Integr Med 2009;7:889-96.

16 Doi SA, Barendregt JJ, Khan S, et al. Advances in the meta-analysis of heterogeneous clinical trials I: the inverse variance heterogeneity model. Contemp Clin Trials 2015:45:130-8.

17 Broeg-Morvay A, Mordasini P, Bernasconi C, et al. Direct mechanical intervention versus combined intravenous and mechanical intervention in large artery anterior circulation stroke: a matched-pairs analysis. Stroke 2016;47:1037-44.

18 Wang H, Zi W, Hao Y, et al. Direct endovascular treatment: an alternative for bridging therapy in anterior circulation large-vessel occlusion stroke. Eur I Neurol 2017:24:935-43.

19 Weber R, Nordmeyer H, Hadisurya J, et al. Comparison of outcome and interventional complication rate in patients with acute stroke treated with mechanical thrombectomy with and without bridging thrombolysis. J Neurointerv Surg 2017;9:229-33.

20 Coutinho JM, Liebeskind DS, Slater LA, et al. Combined intravenous thrombolysis and thrombectomy vs thrombectomy alone for acute ischemic stroke: a pooled analysis of the SWIFT and STAR studies. JAMA Neurol 2017;74:268-74.

21 Abilleira S, Ribera A, Cardona P, et al. Outcomes after direct thrombectomy or combined intravenous and endovascular treatment are not different. Stroke 2017:48:375-8.

22 Guedin P, Larcher A, Decroix JP, et al. Prior IV thrombolysis facilitates mechanical thrombectomy in acute ischemic stroke. J Stroke Cerebrovasc Dis 2015;24:952-7.

23 Maier IL, Behme D, Schnieder M, et al. Bridging-therapy with intravenous recombinant tissue plasminogen activator improves functional outcome in patients with endovascular treatment in acute stroke. J Neurol Sci 2017;372:300-4

24 Rai AT, Boo S, Buseman C, et al. Intravenous thrombolysis before endovascular therapy for large vessel strokes can lead to significantly higher hospital costs without improving outcomes. J Neurointerv Surg 2018;10:17-21.

25 Leker RR, Pikis S, Gomori JM, et al. Is bridging necessary? A pilot study of bridging versus primary stentriever-based endovascular reperfusion in large anterior circulation strokes. J Stroke Cerebrovasc Dis 2015;24:1163-7.

26 Minnerup J, Wersching H, Teuber A, et al. Outcome after thrombectomy and intravenous thrombolysis in patients with acute ischemic stroke: a prospective observational study. Stroke 2016;47:1584-92.

27 Alonso de Leciñana M, Martínez-Sánchez P, García-Pastor A, et al. Mechanical thrombectomy in patients with medical contraindications for intravenous thrombolysis: a prospective observational study. J Neurointerv Surg 2017;9:1041-6.

28 Dávalos A, Pereira VM, Chapot $R$, et al. Retrospective multicenter study of Solitaire FR for revascularization in the treatment of acute ischemic stroke. Stroke 2012;43:2699-705 
29 Nogueira RG, Zaidat 00, Castonguay AC, et al. Rescue thrombectomy in large vessel occlusion strokes leads to better outcomes than intravenous thrombolysis alone: a 'Real World' applicability of the recent trials. Interv Neurol 2016;5(3-4):101-10.

30 Mulder MJ, Berkhemer OA, Fransen PS, et al. Treatment in patients who are not eligible for intravenous alteplase: MR CLEAN subgroup analysis. Int I Stroke 2016:11:637-45

31 Sanak D, Skoloudik D, Veverka T, et al. Prior IV thrombolysis has probably no impact on early recanalization and clinical outcome after endovascular treatment of the occluded cerebral artery in acute ischemic stroke patients. Cerebrovasc Dis 2013:35:178.

32 Kaesmacher J, Kleine JF. Bridging therapy with i.v. rtPA in MCA occlusion prior to endovascular thrombectomy: a double-edged sword? Clin Neuroradiol 2018;28:81-9.

33 Behme D, Kabbasch C, Kowoll A, et al. Intravenous thrombolysis facilitates successful recanalization with stent-retriever mechanical thrombectomy in middle cerebral artery occlusions. J Stroke Cerebrovasc Dis 2016:25:954-9.

34 Mistry AM, Mistry EA. Considerations in meta-analyses to understand the value of intravenous thrombolysis in current, guideline-based, endovascular practice of stroke treatment. World Neurosurg 2017; 108:961.

35 Desilles JP, Loyau S, Syvannarath V, et al. Alteplase reduces downstream microvascular thrombosis and improves the benefit of large artery recanalization in stroke. Stroke 2015;46:3241-8.

36 Kaesmacher J, Boeckh-Behrens T, Simon S, et al. Risk of thrombus fragmentation during endovascular stroke treatment. AJNR Am J Neuroradiol 2017;38:991-8.

37 Almekhlafi MA, Mishra S, Desai JA, et al. Not all 'successful' angiographic reperfusion patients are an equal validation of a modified $\mathrm{TICI}$ scoring system. Interv Neuroradiol 2014;20:21-7.

38 Dargazanli C, Consoli A, Barral M, et al. Impact of modified $\mathrm{TICI} 3$ versus modified $\mathrm{TICl}$ $2 \mathrm{~b}$ reperfusion score to predict good outcome following endovascular therapy. AJNR Am J Neuroradiol 2017;38:90-6.

39 Kleine JF, Wunderlich S, Zimmer C, et al. Time to redefine success? $\mathrm{TICI} 3$ versus TIC $2 \mathrm{~b}$ recanalization in middle cerebral artery occlusion treated with thrombectomy. $J$ Neurointerv Surg 2017;9:117-21.

40 Tung EL, McTaggart RA, Baird GL, et al. Rethinking thrombolysis in cerebral infarction 2b. Stroke 2017:48:2488-93.

41 Kaesmacher J, Maegerlein C, Zibold F, et al. Improving mTICI2b reperfusion to $\mathrm{mTICI} 2 \mathrm{C} / 3$ reperfusions: a retrospective observational study assessing technical feasibility, safety and clinical efficacy. Eur Radiol 2018;28:274-82.

42 Kaesmacher J, Dobrocky T, Heldner MR, et al. Systematic review and meta-analysis on outcome differences among patients with $\mathrm{TICI} 2 \mathrm{~b}$ versus $\mathrm{TICI} 3$ reperfusions: success revisited. J Neurol Neurosurg Psychiatry 2018. doi: 10.1136/jnnp-2017-317602. [Epub ahead of print].

43 Seet RC, Zhang Y, Wijdicks EF, et al. Relationship between chronic atrial fibrillation and worse outcomes in stroke patients after intravenous thrombolysis. Arch Neurol 2011;68:1454-8.

44 Steger C, Pratter A, Martinek-Bregel M, et al. Stroke patients with atrial fibrillation have a worse prognosis than patients without: data from the Austrian Stroke registry. Eur Heart J 2004;25:1734-40.
45 Marini C, De Santis F, Sacco S, et al. Contribution of atrial fibrillation to incidence and outcome of ischemic stroke: results from a population-based study. Stroke 2005;36:1115-9.

46 Arnold M, Halpern M, Meier N, et al. Age-dependent differences in demographics, risk factors, co-morbidity, etiology, management, and clinical outcome of acute ischemic stroke. J Neuro/ 2008:255:1503-7.

47 Saver JL, Goyal M, van der Lugt A, et al. Time to Treatment With Endovascula Thrombectomy and Outcomes From Ischemic Stroke: A Meta-analysis. JAMA 2016:316:1279.

48 Fiorelli M, Bastianello S, Kummer V, et al. Hemorrhagic transformation within 36 hours of a cerebral infarct. Stroke 1999:30:2280-4.

49 Kaesmacher J, Kaesmacher M, Maegerlein C, et al. Hemorrhagic transformations after thrombectomy: risk factors and clinical relevance. Cerebrovasc Dis 2017:43:294-304

50 Hao $\mathrm{Y}$, Yang D, Wang $\mathrm{H}$, et al. Predictors for symptomatic intracranial hemorrhage after endovascular treatment of acute ischemic stroke. Stroke 2017:48:1203-9.

51 Nogueira RG, Gupta R, Jovin TG, et al. Predictors and clinical relevance of hemorrhagic transformation after endovascular therapy for anterior circulation large vesse occlusion strokes: a multicenter retrospective analysis of 1122 patients. J Neurointerv Surg 2015;7:16-21.

52 Wang DT, Churilov L, Dowling R, et al. Successful recanalization post endovascular therapy is associated with a decreased risk of intracranial haemorrhage: a retrospective study. BMC Neurol 2015;15:185

53 Emberson J, Lees KR, Lyden $\mathrm{P}$, et al. Effect of treatment delay, age, and stroke severity on the effects of intravenous thrombolysis with alteplase for acute ischaemic stroke: a meta-analysis of individual patient data from randomised trials. Lancet 2014:384:1929-35.

54 Kwiatkowski TG, Libman RB, Frankel M, et al. Effects of tissue plasminogen activator for acute ischemic stroke at one year. N Eng/ J Med 1999:340:1781-7.

55 Menon BK, Najm M, Al-Ajlan F, et al. Abstract 186: IV tPA recanalization rates by site of occlusion and time after tpa bolus- main results of the interrsect multinational multicenter prospective cohort study. Stroke 2017;48:186.

56 Berkhemer OA, Fransen PSS, Beumer D, et al. A randomized trial of intraarterial treatment for acute ischemic stroke. N Engl J Med 2015;372:11-20.

57 Mao YT, Mitchell P, Churilov L, et al. Early recanalization postintravenous thrombolysis in ischemic stroke with large vessel occlusion: a digital subtraction angiography study. CNS Neurosci Ther 2016;22:643-7.

58 Behrens L, Möhlenbruch M, Stampfl S, et al. Effect of thrombus size on recanalization by bridging intravenous thrombolysis. Eur J Neurol 2014;21:1406-10.

59 Mueller L, Pult F, Meisterernst J, et al. Impact of intravenous thrombolysis on recanalization rates in patients with stroke treated with bridging therapy. Eur J Neurol 2017; 24:1016-21.

60 Tsivgoulis G, Katsanos AH, Schellinger PD, et al. Successful reperfusion with intravenous thrombolysis preceding mechanical thrombectomy in large-vesse occlusions. Stroke 2018:49:232-5.

61 Wee CK, McAuliffe W, Phatouros CC, et al. Outcomes of Endovascular Thrombectomy with and without Thrombolysis for Acute Large Artery Ischaemic Stroke at a Tertiary Stroke Centre. Cerebrovasc Dis Extra 2017;7:95-102. 